\title{
Stable Angina Treatment Strategies and Current Practices in Lahore, Pakistan: A Cross-Sectional Analysis
}

\author{
*Ali Ahmed ${ }^{1,2}$, Maria Tanveer ${ }^{1,3}$, Naheed Bano ${ }^{2}$ and Gul Majid Khan ${ }^{1}$ \\ ${ }^{1}$ Quaid-i-Azam University, Islamabad 45320, Pakistan \\ ${ }^{2}$ University of veterinary and animal sciences, Lahore, Pakistan \\ ${ }^{3}$ University of Lahore, Lahore, Pakistan
}

\begin{abstract}
The purpose of the study was to analyze the prescription trends in the treatment of stable angina and to identify the therapeutic agents being prescribed majorly In Pakistan and to analyze that whether the current prescription patterns are in accordance with evidence based guidelines. A concurrent type of study was conducted from 24-01-2015 to 24-04-2015, through collection of data from prescriptions of patients suffering from stable angina in public sector hospitals of Lahore Pakistan. Data observations from the total 200 prescriptions collected from the hospitals of Lahore graphically demonstrate the trends of drug classes per prescription. The study identified that in the treatment of stable angina Antiplatelet are the most frequently prescribed drug class in Lahore and adjacent areas. Followed by CCBs on $2^{\text {nd }}$ rank and nitrates on $3^{\text {rd }}$ rank, ACE inhibitors and ARBs on $4^{\text {th }}$ number, after which statins occupy the $5^{\text {th }}$ position, lastly comes the position of beta blocker i.e. $6^{\text {th }}$ and diuretics are the least prescribed class. The anti-platelet blocker drugs were the most prescribed drug class in the management of chronic stable angina followed by calcium channel and vasodilators occupying the second and third rank respectively. The study also revealed that men are 1.5 times more vulnerable to the development of angina than the females. The study is also showing concrete evidence that there is strong need to follow guidelines for proper treatment.
\end{abstract}

Key Words: Stable Angina, Vasodilators, Treatment strategies, Nitrates.

\section{INTRODUCTION}

Stable chronic angina is an ischemic heart disease defined as a medical condition characterized by the symptoms of chest pain, sensation of pressure, pain in jaws, left shoulder, left arm, over the sternum, lower cervical and upper thoracic (Bukhsh et al., 2014). Chronic stable angina has a predictable course of occurrence, predominantly, associated with exertion or emotional events culminating in a transient myocardial ischemia resulting in the precipitation of sensations of pressure or hard squeezing, vaguely localized, in the sub-sternal chest region. Emotional stress and moderate to severe physical activity can also demonstrate the precipitation of equivalents of angina including breathlessness associated with exertion, malaise and fatigue. The usual etiology of angina is myocardial ischemia resulting from a myocardium demand and supply imbalance for oxygen (Abrams and Thadani, 2005). In turn, myocardial ischemia is a result of coronary atherosclerosis (Davies, 2001). Temperature is also an exaggerating factor of chest pain. Cold environment may induce chest pain due to reduction in the blood supply and vasoconstriction (Bukhsh et al., 2014). Angina is also precipitated by certain uncommon or rare events including congenital anomalies, hypertrophic cardiac myopathy and some physiological states requiring high output like severe anemia. Almost half of the imminent angina cases are not recognized through risk factor identification but are reported once chest pain precipitates (Davies, 2001) .

The evaluation of prescribing trends in ambulatory care cardiac patients above 50 years of age in Lahore reveal;
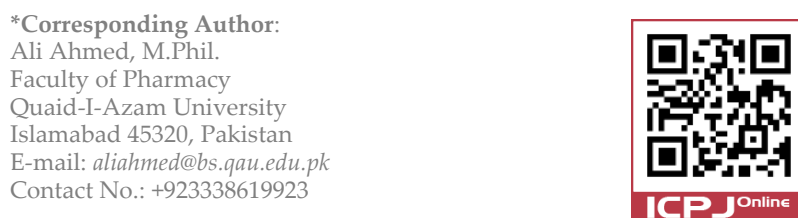

the prevalence of the Ischemic heart disease in Lahore hospitals is $35.7 \%$, which is higher compared to the other cardiac diseases. In addition, mortality rate due to cardiovascular problems is $85 \%$ in developing countries and observed that cardiovascular diseases arise mostly due to co-morbid conditions. The most prevalent co- morbidities are HTN $(47.7 \%)$, DM $(40.5 \%)$, Hyperlipidemias $(6.3 \%)$, COPD $(3.6 \%)$, hepatitis $(0.9 \%)$, pulmonary edema $(0.9 \%)$ and case of IHD the mainly are HTN (29\%) and DM (19\%) (Bukhsh et al., 2014).

The mortality rate associated with angina is $1.6-3.2 \%$ annually. In both sexes, increasing age is a risk factor for SA. In reference to age occurrence of angina, 45-54 years $(0.1-1 \%)$ at age $65-74(10-15 \%)$ in women and in men at $45-$ $54(2-5 \%)$ and at $65-74(2-5 \%)$. Hence, estimation shows that almost 20000-40000 people per million die due to angina pectoris.

Given the current rise of angina in Pakistan, this study was designed to evaluate the prescribing trends of stable angina and to find out the most prescribed drug in stable angina in public sector hospitals Lahore, Pakistan and assessing its prevalence in both genders.

\section{METHODOLOGY}

A concurrent type of study was conducted for 3 months from 24-01-2015 to 24-04-2015, through collection of data from prescriptions of patients suffering from stable angina in public sector hospitals of Lahore Pakistan (Punjab Institute of Cardiology \& Shaikh Zayed Hospital). The Purpose of such data collection was to study the most prescribed drug in the treatment of stable angina, prevalence of angina among different genders. 200 prescriptions were collected and studied for the drugs prescribed, major parameter of study was the prescribing trends of stable angina. Statistical analysis of data was performed using SPSS 20 and results are plotted in form of bar and pie charts. 


\section{Inclusion Criteria}

The OPD patients were randomly selected with an age group of greater than 35 years and both genders were given equal preference.

\section{Exclusion Criteria}

The patients having post bypass angina were excluded as well as those patients that had prinzmetal and unstable angina.

\section{Study Variables}

Medication profile of each patient containing complete prescription info was studied along with Medical Service File (containing info about type of visits, hospital testing record and info of the drug prescribed). In addition, further information was also gathered by verbally questioning patients.

\section{RESULTS}

The overall graphical presentation of drugs according to their percentages is represented in figure 1 . The pie charts Figure 2 to 6 illustrate the results of the study revealing information about usage of each drug of each prescribed class among the study population.

\section{Vasodilators}

Among vasodilators class $77 \%$ GTN is prescribed, while Isosorbide is prescribed $10 \%$, combination of Isosorbide mononitrate and GTN is also being prescribed $10 \%$ while diluted Nitroglycerine is prescribed 3\% in the public-sector hospitals of localities of Lahore, Pakistan, figure 2.

\section{Beta Blockers (BB)}

The data indicates that Metoprolol (45\%) is the drug of choice in this class while Bisoprolol $(21 \%)$ is the second most prescribed drug. Atenolol $(18 \%)$ is the third most choice of physicians, Carvedilol $(11 \%)$ is forth on rank. In combination Bisoprolol and metoprolol show 3\% trend of prescription. Propranolol individually and in combination of Metoprolol and carvedilol show same trends of prescribing i.e. $1 \%$, figure 3 .

\section{Antiplatelet Drugs}

In this class, Aspirin is at the top of prescription with trend of $45 \%$. While combination of aspirin and Clopidogril also shows almost equal trend with a percentage of $44 \%$. Clopidogril is the least prescribed antiplatelet with $11 \%$, figure 4 .

\section{Anihyperlipidemics (statins)}

Among Antihyperlipidemics, only statins are being prescribed in publics sector hospitals of Lahore. Atorvastatin is the most prescribed drug with $75 \%$, Rosuvastatin on second with $20 \%$ and Simvastatin with 5\% trends in prescriptions in public sector Hospitals of Lahore, figure 5.

\section{Calcium Channel Blockers (CCBs)}

Calcium channel blockers are usually prescribed in comorbid conditions in which beta blockers cannot be prescribed like COPD, patients on Digoxin therapy etc. Compared with beta blockers Calcium channel blockers are of less use in South Asia. In Lahore CCBs are prescribed in percentages as Amlodipine $87 \%$ and Nifedepine with percentage of $13 \%$, figure 6 .

\section{ACE Inhibitors/Angiotensin Receptor Blockers}

Drugs under this class are further combination of two subclasses, however collectively the percentage of drugs of this class is Lisinopril 47\%, Losartan 44\%, Captopril 6\% and Enalapril with percentage of $3 \%$, figure 7 .

\section{Diuretics}

Diuretics are the backbone of treatment in maximum patients with cardiovascular diseases. The Frequency of drugs among this class is as, Furosemide 63\%, Spironolactone $23 \%$, Combination of Spironolactone with Furosemide is also surpassing many sole drugs with percentage of $9 \%$, Hydrochlorothiazide 3\% and Amiloride with 2\%, figure 8 .

\section{Prevalence of Stable Angina in Male vs Females}

In Literature, Studies revealed that men are at more risk of stable angina than women are, while prognosis of disease is more severe and complex in females than males. The study-designed data graphically shows that in population of Lahore, Pakistani men are more vulnerable to occurrence of angina than women are. The percentage of men is $60 \%$ which is more than that of women i.e. $40 \%$, figure 9 .

\section{DISCUSSION}

In Lahore, an important city of Pakistan, cardiovascular diseases are prevalent due to malnutrition, sluggish life style and also due to increasing psychological stress. Occurrence of chest pain is the alarming situation indicating angina, and when untreated, lead to USA or MI. Optimum therapy for stable angina involves ruling out the risk factors of MI to a significant extent. Numerous guidelines of treatment are available to ensure that prescribers follow optimum therapy and practice safe use of therapeutic resources. American Heart Association (AHA) and European Clinical Society (ECS) are among such bodies that are continuously optimizing the therapy by standardizing the guidelines. Data observations from the total 200 prescriptions collected from the hospitals of Lahore graphically demonstrate the trends of drug classes per prescription. The study identified that in the treatment of stable angina Antiplatelet are the most frequently prescribed drug class in Lahore and adjacent areas. Followed by CCBs on $2^{\text {nd }}$ rank and nitrates on $3^{\text {rd }}$ rank, ACE inhibitors and ARBs on $4^{\text {th }}$ number, after which statins occupy the $5^{\text {th }}$ position, lastly comes the position of beta blocker i.e., $6^{\text {th }}$ and diuretics are the least prescribed class.

An effective treatment of angina requires the utilization of evidence based guidelines to guide therapeutic decisions. A single appropriate selection from the armamentarium can pave way for optimal therapeutic outcomes. As far as the issue of Evidence based treatment of angina is concerned, prospective studies have shown that aggressive blood pressure control and serum LDL level optimization alone can produce paramount effects in angina incidence reduction (Tobin, 2010). The JNC-8 Guidelines recommend the use Thiazide Diuretics as the initial line of therapeutic regimen. (James et al., 2014). However, our study reveals that of the 200 prescriptions analyzed, only 59 prescribed Diuretics. Thus, only one fourth of the patients actually received an optimal standardized therapeutic regimen. The rest of the three quarters failed to receive blood pressure control treatment as recommended by the JNC-8 Guidelines.

The study also revealed that the occurrence of Stable angina is more prevalent amongst males accounting for $60 \%$ of the total cases, whereas, females demonstrate an incidence rate of $40 \%$. Hence, men are at more risk of development of SA than women. 


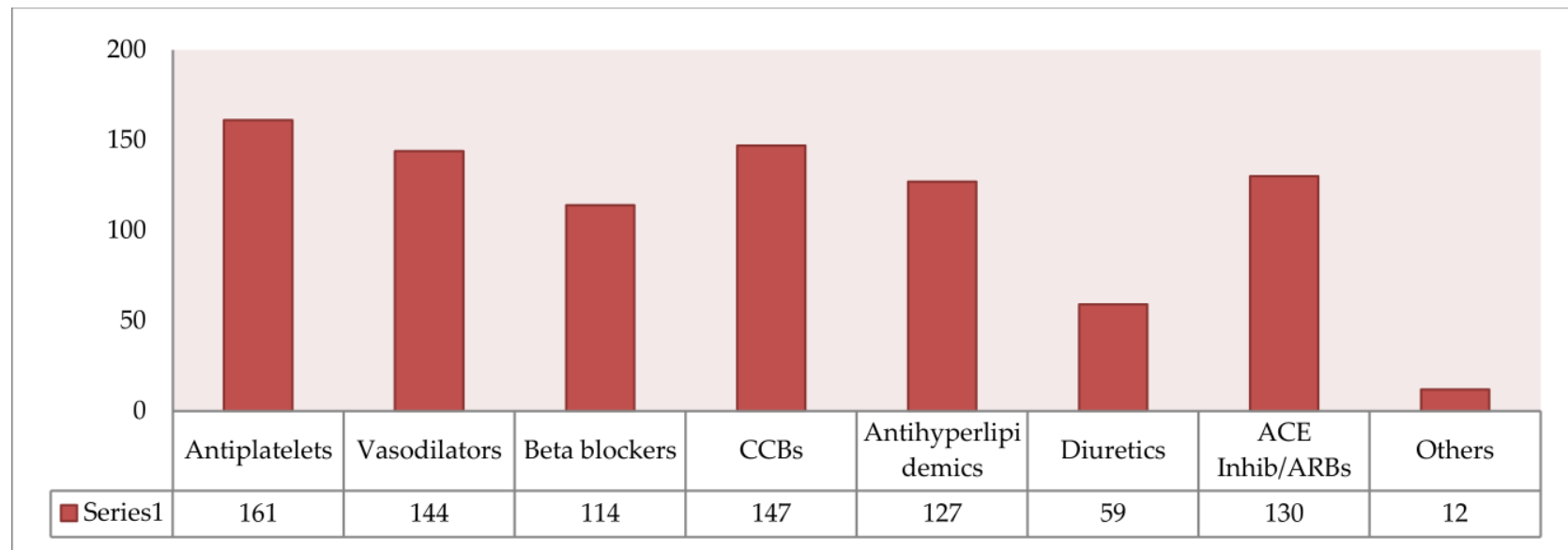

Figure 1: Percentage of each class of drug according to their trend in prescription.

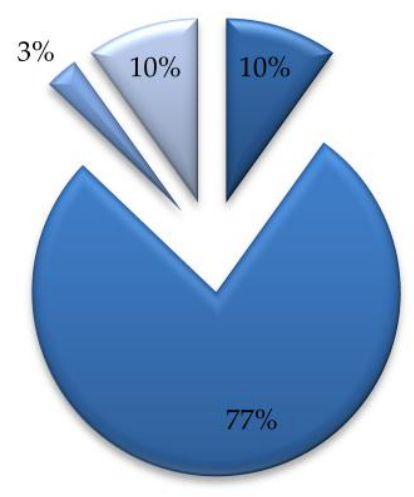

घisosorbiidemononitrate 口GTN

Figure 2: Prescription trends for Vasodilators.

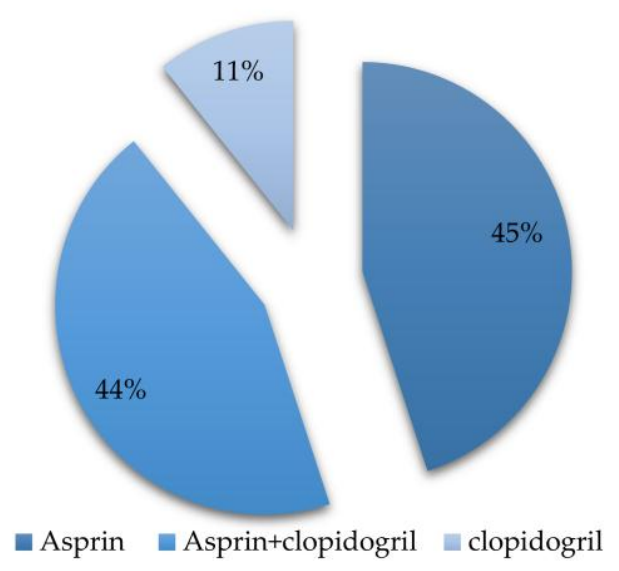

Figure 4: Prescribing trends of antiplatelets.
$3 \% 1 \%$

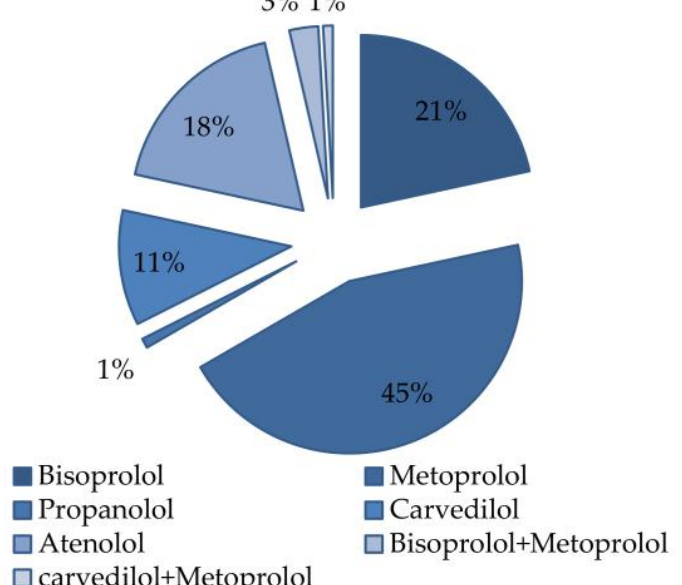

Figure 3: Prescribing trends of beta blockers.

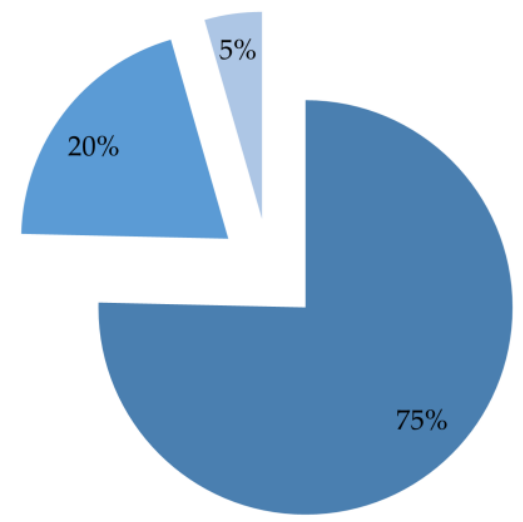

Atorvastation Rosuvastatin Simvastatin

Figure 5: Trends of antihyperlipidemics. 


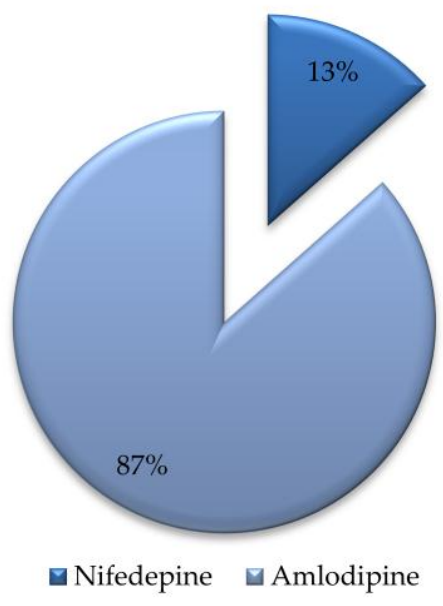

Figure 6: Prescribing trends of calcium channel blockers.

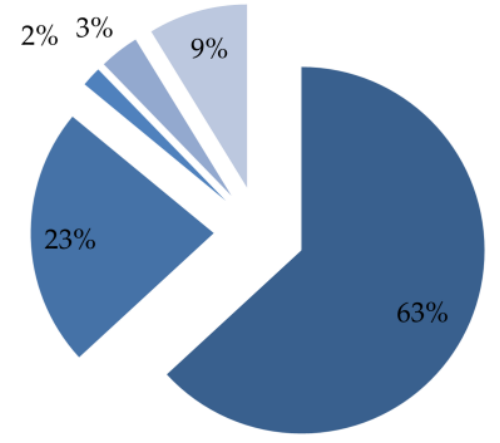

Furosemide

- Spironolacrones

Figure 8: Prescribing trends of diuretics.

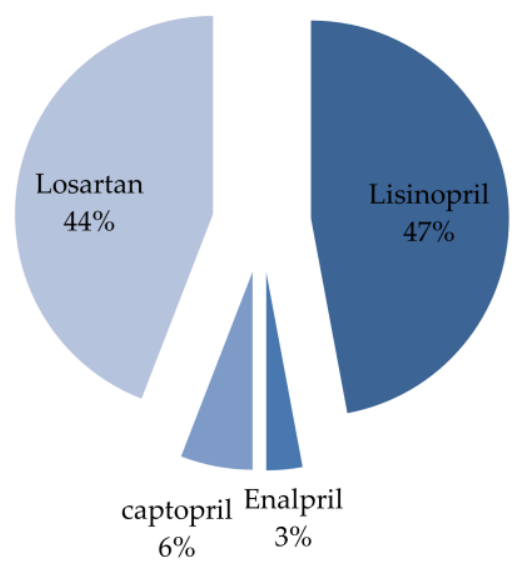

Figure 7: Prescribing Trends of ACE Inhibitors and ARBs.

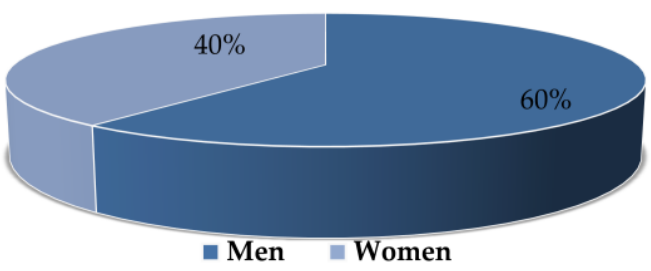

Figure 9: Comparison of Prevalence of Angina in Males and Females. 


\section{CONCLUSION}

The class of drug that is most frequently prescribed in Stable Angina in health care sectors of Lahore Pakistan is Anti platelet class of Drugs, among which Aspirin is the most prescribed drug. Moving away from standard guidelines, risk of SA is increasing with age and men are at more risk of SA. For optimal therapy and safe use of medicine, at least one beta blocker should be added in the therapy. Males above 35 with increased serum lipids level should regularly check their BP and should avoid fatty diet and should take steps to regulate their plasma triglycerides level.

\section{REFERENCES}

Abrams, J., Thadani, U., (2005). Therapy of stable angina pectoris. The Uncomplicated Patient, 112(15), e255-e259. [DOI]

Bukhsh, A., Saif, S., Imtiaz, H., Arif, I., Muzaffar, A., Saleem, R., (2014). Evaluation of prescribing trends in ambulatory cardiac patients above 50 years of age in lahore, pakistan.

Davies, S.W., (2001). Clinical presentation and diagnosis of coronary artery disease: Stable angina. British Medical Bulletin, 59(1), 17-27. [DOI]

James, P.A., Oparil, S., Carter, B.L., Et Al., (2014). 2014 evidence-based guideline for the management of high blood pressure in adults: Report from the panel members appointed to the eighth joint national committee (jnc 8). JAMA, 311(5), 507-520. [DOI]

Tobin, K.J., (2010). Stable angina pectoris: What does the current clinical evidence tell us? The Journal of the American Osteopathic Association, 110(7), 364-370. 